\title{
The Beta Exponential Fréchet Distribution with Applications
}

\author{
M. E. Mead \\ Ahmed Z. Afify \\ G. G. Hamedani \\ Indranil Ghosh \\ Zagazig University \\ Benha University \\ Marquette University \\ UNCW
}

\begin{abstract}
We define and study a new generalization of the Fréchet distribution called the beta exponential Fréchet distribution. The new model includes thirty two special models. Some of its mathematical properties, including explicit expressions for the ordinary and incomplete moments, quantile and generating functions, mean residual life, mean inactivity time, order statistics and entropies are derived. The method of maximum likelihood is proposed to estimate the model parameters. A small simulation study is also reported. Two real data sets are applied to illustrate the flexibility of the proposed model compared with some nested and non-nested models.
\end{abstract}

Keywords: generating function, maximum likelihood, entropy, Fréchet distribution, beta exponential-G family.

\section{Introduction}

In the past few decades, many generators have been proposed by extending some useful statistical distributions. Such generated families of distributions have been extensively used for modeling and analyzing lifetime data in many applied sciences such as reliability, engineering, actuarial sciences, demography, economics, hydrology, biological studies, insurance, medicine and finance, among others. However, there still remain many real world phenomena involving data, which do not follow any of the classical statistical distributions.

The Fréchet distribution was proposed to model extreme events such as annually maximum one-day rainfalls and river discharges by Fréchet (1924). This distribution has found wide application in extreme value theory. Further details about the Fréchet distribution can be found in Kotz and Nadarajah (2000).

Some extensions of the Fréchet distribution are available in the literature, such as the exponentiated Fréchet (EFr) (Nadarajah and Kotz, 2003), beta Fréchet (BFr) (Nadarajah and Gupta, 2004 and Barreto-Souza et al., 2011), transmuted Fréchet (TFr) (Mahmoud and Mandouh, 2013), gamma extended Fréchet (GEFr) (Silva et al., 2013), Marshall-Olkin Fréchet (Krishna et al., 2013), transmuted exponentiated Fréchet (TEFr) (Elbatal et al., 2014), Kumaraswamy Fréchet (Kw-Fr) (Mead and Abd-Eltawab, 2014), transmuted Marshall-Olkin 
Fréchet (TMOFr) (Afify et al., 2015a) and Weibull Fréchet (WFr) (Afify et al., 2016).

The cumulative distribution function (cdf) and probability density function (pdf) of the Fréchet (Fr) distribution are, respectively, given by (for $x>0$ )

$$
G_{\mathrm{Fr}}(x ; \theta, \beta)=e^{-\left(\frac{\theta}{x}\right)^{\beta}} \text { and } g_{\mathrm{Fr}}(x ; \theta, \beta)=\beta \theta^{\beta} x^{-\beta-1} e^{-\left(\frac{\theta}{x}\right)^{\beta}},
$$

where $\theta>0$ is a scale parameter and $\beta>0$ is a shape parameter, respectively.

The aim of this paper is to provide another extension of the Fréchet model using the Beta exponential-G (BEx-G) family of distributions proposed by Alzaatreh et al. (2013). So, we propose the new beta exponential Fréchet (BExFr for short) distribution by adding three extra shape parameters to the Fréchet distribution. The objective of this work is to study some mathematical properties of the five-parameter BExFr model with the hope that it will attract wider applications in reliability, engineering and other areas of research.

For an arbitrary baseline cdf $G(x)$, Alzaatreh et al. (2013) defined the BEx-G family of distributions by the cdf and pdf

$$
F(x ; a, b, \lambda)=\frac{1}{B(a, b)} B\left(1-(1-G(x))^{\lambda} ; a, b\right)
$$

and

$$
f(x ; a, b, \lambda)=\frac{\lambda g(x)}{B(a, b)}[1-G(x)]^{\lambda b-1}\left\{1-[1-G(x)]^{\lambda}\right\}^{a-1},
$$

respectively, where $g(x)=d G(x) / d x$ and $a, b$ and $\lambda$ are three extra positive shape parameters, $B(z ; a, b)=\int_{0}^{z} t^{a-1}(1-t)^{b-1} d t$ is the incomplete beta function, $B(a, b)=\Gamma(a) \Gamma(b) / \Gamma(a+b)$ and $\Gamma($.$) is the gamma function. Clearly, when a=b=\lambda=1$, we obtain the baseline distribution. If $X$ is a random variable with pdf (3), we write $X \sim \operatorname{BEx}-\mathrm{G}(a, b, \lambda)$. An attractive feature of this model is that these parameters can afford greater control over the weights in both tails and in its center.

Next, we consider the Fr model in order to define the new distribution by taking $G(x)$ in $(2)$ to be the cdf in (1) of the Fr distribution. Then, the cdf, say $F(x)=F(x ; a, b, \lambda, \beta, \theta)$, of the BExFr distribution (for $x>0$ ) reduces to

$$
F(x)=\frac{1}{B(a, b)} B\left(1-\left(1-e^{-\left(\frac{\theta}{x}\right)^{\beta}}\right)^{\lambda} ; a, b\right) .
$$

The corresponding pdf follows by inserting (1) in equation (3)

$$
f(x)=\frac{\lambda \beta \theta^{\beta}}{B(a, b)} x^{-\beta-1} e^{-\left(\frac{\theta}{x}\right)^{\beta}}\left[1-e^{-\left(\frac{\theta}{x}\right)^{\beta}}\right]^{\lambda b-1}\left\{1-\left[1-e^{-\left(\frac{\theta}{x}\right)^{\beta}}\right]^{\lambda}\right\}^{a-1} .
$$

Henceforth, $X \sim \operatorname{BEx} \operatorname{Fr}(a, b, \lambda, \beta, \theta)$ denotes a random variable having density function (5). The survival function (sf) and hrf of $X$ are, respectively, given by

$$
S(x)=1-\frac{1}{B(a, b)} B\left(1-\left(1-e^{-\left(\frac{\theta}{x}\right)^{\beta}}\right)^{\lambda} ; a, b\right)
$$

and

$$
h(x)=\frac{\lambda \beta \theta^{\beta} x^{-\beta-1} e^{-\left(\frac{\theta}{x}\right)^{\beta}}\left[1-e^{-\left(\frac{\theta}{x}\right)^{\beta}}\right]^{\lambda b-1}\left\{1-\left[1-e^{-\left(\frac{\theta}{x}\right)^{\beta}}\right]^{\lambda}\right\}^{a-1}}{\left\{B(a, b)-B\left(1-\left(1-e^{-\left(\frac{\theta}{x}\right)^{\beta}}\right)^{\lambda} ; a, b\right)\right\}} .
$$

The BExFr distribution appears to have the ability to model failure rate models which are quite common in reliability and biological studies. Furthermore, a possible application of the 
BExFr distribution could be in modeling ordinal data as a latent response models. Ordinal data are commonly used in many areas of application, some examples being the quality of an item or service or performance (poor, fair, good, very good or excellent), seriousness of a defect (minor, major, critical), taste of food (too mild, just right, too spicy) and extent of agreement (strongly disagree, disagree, neutral, agree, strongly agree). Many techniques are available for analyzing stochastic shifts in ordinal data; for a review see Agresti (1984). However, serious difficulties arise when inferences are desired on both location and dispersion effects; see Nair (1986) and Hamada and $\mathrm{Wu}$ (1990) and the accompanying discussions. The main cause of difficulty in separating the location effects from dispersion effects when the data are ordinal is that the number of categories is usually small (between 3 and 10). Therefore, when the location parameter is pushed to the limit (either too high or too low), most of the data fall in the extreme category giving a false impression of reduced variance. A common approach to the analysis of ordinal data is to assume a continuous latent response distribution that is observed through windows of ordered intervals with fixed, but unknown cutpoints. This approach is implicit in the proportional odds model (McCullagh, 1980), which can be derived from an underlying logistic response distribution and in other generalized linear models (McCullagh and Nelder, 1989). These models are typically based on the assumption of a symmetric continuous latent response having an infinite domain.

To resolve these difficulties, we propose the BExFr distribution as a model for the latent variable. The following two properties of the beta distribution make it especially suitable for modeling ordinal data:

- The BExFr distribution has an infinite domain.

- The BExFr distribution can flexibly model a wide variety of shapes including a bellshape (symmetric or skewed), U-shape and J-shape.

The BExFr distribution is a very flexible model having several special cases. It contains 32 sub-models listed in Table 1. The BExFr includes some important sub-models, namely: the beta exponential inverse Rayleigh (BExIR), beta exponential inverse exponential (BExIEx), beta Fréchet (BFr), beta inverse Rayleigh (BIR), beta inverse exponential (BIEx), exponentiated exponential Fréchet (EExFr), exponentiated exponential inverse Rayleigh (EExIR), exponentiated exponential inverse exponential (EExIEx), beta exponential generalized inverse Weibull (BExGIW) and beta exponential generalized inverse Rayleigh (BExGIR) distributions. Figure 1 displays some plots of the BExFr density for some values of the parameters $a, b, \lambda, \beta$ and $\theta$. Further, plots of the hrf of the new distribution are shown in Figure 2.

We provide a comprehensive description of some mathematical properties of the BExFr distribution. The paper is outlined as follows. In Section 2, we derive useful representations for the pdf and cdf of the BExFr. Some mathematical properties including the quantile function (qf), ordinary and incomplete moments, moment generating function (mgf), Rényi, Shannon and q-entropies, mean residual life (MRL) and mean inactivity time (MIT) are discussed in Section 3. In section 4, we consider order statistics for a random sample of size $\mathrm{n}$ drawn from the BExFr distribution. Certain characterizations are presented in Section 5. In Section 6, we obtain the maximum likelihood estimates (MLEs) of the model parameters. Section 7 deals with a small simulation study. In Section 8, the potentiality of the new model is illustrated by means of two applications to two real data sets. Finally, in Section 9, we provide some concluding remarks.

\section{Mixture representation}

In this section, we derive mixture representations for the pdf and cdf of $X$. In order to obtain 


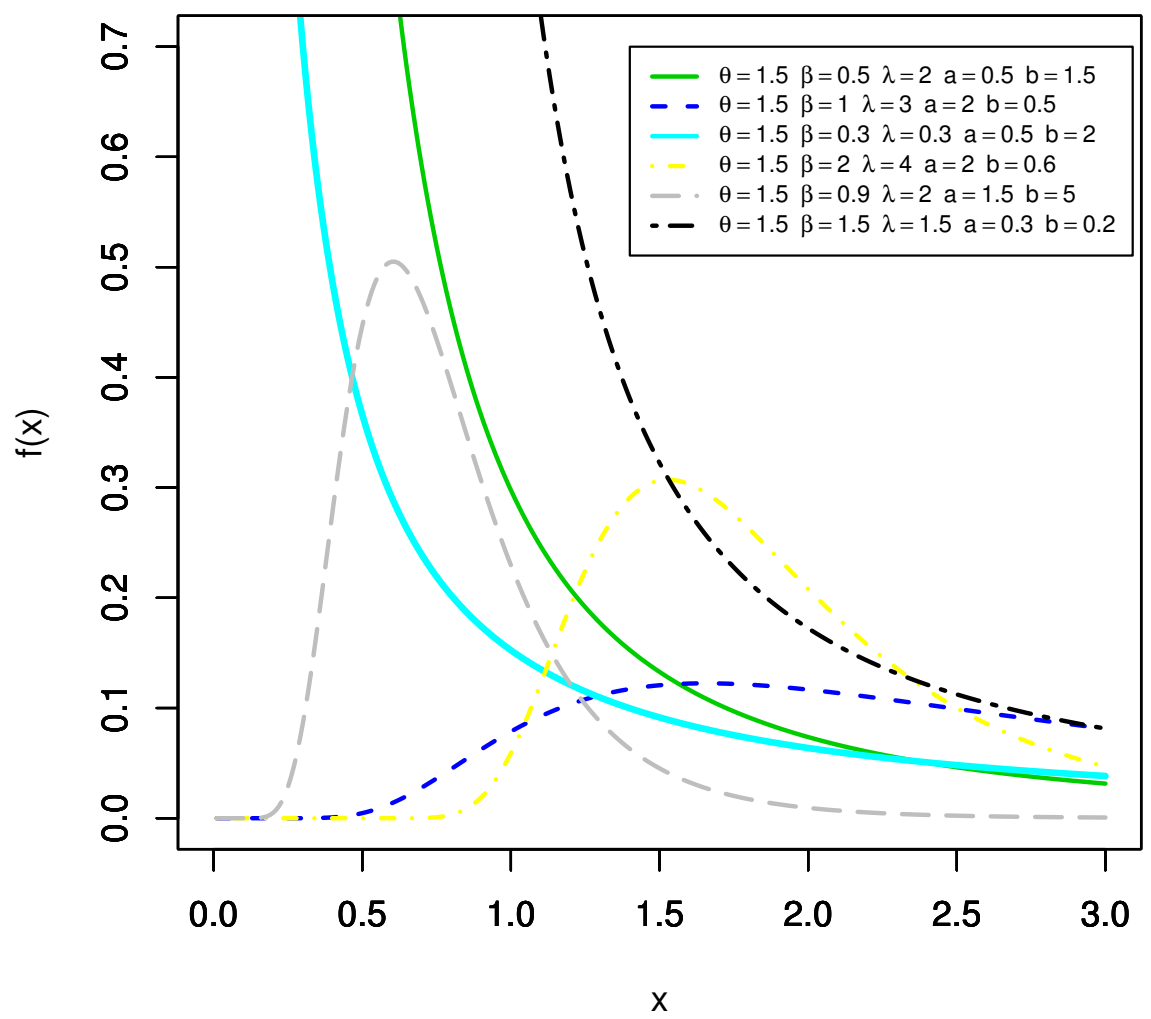

Figure 1: The plots of BExFr density function.

a simple form for the BExFr pdf, we expand (5) using the power series

$$
(1-z)^{b-1}=\sum_{j=0}^{\infty} \frac{(-1)^{j} \Gamma(b)}{j ! \Gamma(b-j)} z^{j},|z|<1, b>0 .
$$

Using expansion (6) in equation (5) and after some algebra, the pdf of $X$ can be written as

$$
f(x)=\frac{\lambda \beta \theta^{\beta}}{B(a, b)} x^{-\beta-1} e^{-\left(\frac{\theta}{x}\right)^{\beta}} \sum_{j=0}^{\infty} \frac{(-1)^{j} \Gamma(a)}{j ! \Gamma(a-j)} \underbrace{\left[1-e^{\left.-\left(\frac{\theta}{x}\right)^{\beta}\right]^{\lambda(b+j)-1}}\right.}_{A} .
$$

By applying (6) in the quantity $A$, the last equation becomes

$$
\begin{gathered}
f(x)=\sum_{k=0}^{\infty} v_{k} \beta(k+1) \theta^{\beta} x^{-\beta-1} e^{-(k+1)\left(\frac{\theta}{x}\right)^{\beta}}, \\
v_{k}=\sum_{j=0}^{\infty} \frac{(-1)^{j+k} \lambda \Gamma(a) \Gamma(\lambda(b+j))}{j !(k+1) B(a, b) \Gamma(a-j) \Gamma(\lambda(b+j)-k)} .
\end{gathered}
$$

Equation (7) can be rewritten as

$$
f(x)=\sum_{k=0}^{\infty} v_{k} h_{k+1}(x),
$$

where $h_{k+1}(x)$ is the Fréchet pdf with shape parameter $\beta$ and scale parameter $\theta(k+1)^{1 / \beta}$. 


\begin{tabular}{|c|c|c|c|c|c|c|}
\hline Reduced & \multicolumn{5}{|c|}{ Parameters } & \multirow{2}{*}{ Author } \\
\cline { 2 - 6 } Model & $\theta$ & $\beta$ & $a$ & $b$ & $\lambda$ & New \\
\hline BExIR & - & 2 & - & - & - & New \\
\hline BExIEx & - & 1 & - & - & - & - \\
\hline BFr & - & - & - & - & 1 & Nadarajah and Gupta (2004) \\
\hline BIR & - & 2 & - & - & 1 & - \\
\hline BIEx & - & 1 & - & - & 1 & New \\
\hline EExFr & - & - & 1 & - & - & New \\
\hline EExIR & - & 2 & 1 & - & - & New \\
\hline EExIEx & - & 1 & 1 & - & - & - \\
\hline EFr & - & - & 1 & - & 1 & Nadarajah and Kotz (2003) \\
\hline EIR & - & 2 & 1 & - & 1 & - \\
\hline EIEx & - & 1 & 1 & - & 1 & New \\
\hline ExFr & - & - & 1 & 1 & - & New \\
\hline ExIR & - & 2 & 1 & 1 & - & New \\
\hline ExIEx & - & 1 & 1 & 1 & - & Fréchet (1924) \\
\hline Fr & - & - & 1 & 1 & 1 & Trayer (1964) \\
\hline IR & - & 2 & 1 & 1 & 1 & New \\
\hline IEx & - & 1 & 1 & 1 & 1 & Keller and Kamath (1982) \\
\hline BExGIW & $q c^{1 / \beta}$ & - & - & - & - & New \\
\hline BExGIR & $q c^{1 / 2}$ & 2 & - & - & - & New \\
\hline BExGIEx & $q c$ & 1 & - & - & - & - \\
\hline BGIW & $q c^{1 / \beta}$ & - & - & - & 1 & Baharith et al. (2014) \\
\hline BGIR & $q c^{1 / 2}$ & 2 & - & - & 1 & - \\
\hline BGIEx & $q c$ & 1 & - & - & 1 & - \\
\hline EExGIW & $q c^{1 / \beta}$ & - & 1 & - & - & New \\
\hline EExGIR & $q c^{1 / 2}$ & 2 & 1 & - & - & New \\
\hline EExGIEx & $q c$ & 1 & 1 & - & - & - \\
\hline EGIW & $q c^{1 / \beta}$ & - & 1 & - & 1 & - \\
\hline EGIR & $q c^{1 / 2}$ & 2 & 1 & - & 1 & New \\
\hline EGIEx & $q c$ & 1 & 1 & - & 1 & de Gusmao et al. (2011) \\
\hline GIW & $q c^{1 / \beta}$ & - & 1 & 1 & 1 & - \\
\hline GIR & $q c^{1 / 2}$ & 2 & 1 & 1 & 1 & $q$ \\
\hline GIEx & $q c$ & 1 & 1 & 1 & 1 & - \\
\hline & & & & & - \\
\hline
\end{tabular}

Table 1: Sub-models of the BExFr distribution.

Equation (8) reveals that the BExFr density function can be expressed as a mixture of Fréchet densities. So, several of its structural properties can be derived from those of the Fréchet distribution.

By integrating (8), we obtain

$$
F(x)=\sum_{k=0}^{\infty} v_{k} H_{k+1}(x)
$$

where $H_{k+1}(x)$ is the cdf of the Fréchet model with shape parameter $\beta$ and scale parameter $\theta(k+1)^{1 / \beta}$.

\section{Mathematical properties}

Established algebraic expansions to determine some structural quantities of the BExFr distribution can be more efficient than computing those directly by numerical integration of its density function.

Let $X$ and $Y$ be two random variables. $X$ is said to be stochastically greater than or equal to $Y$, denoted by $X \underset{\text { st }}{\geqslant} Y$ if $P(X>x) \geq P(Y>x)$ for all $x$ in the support set of $X$. 


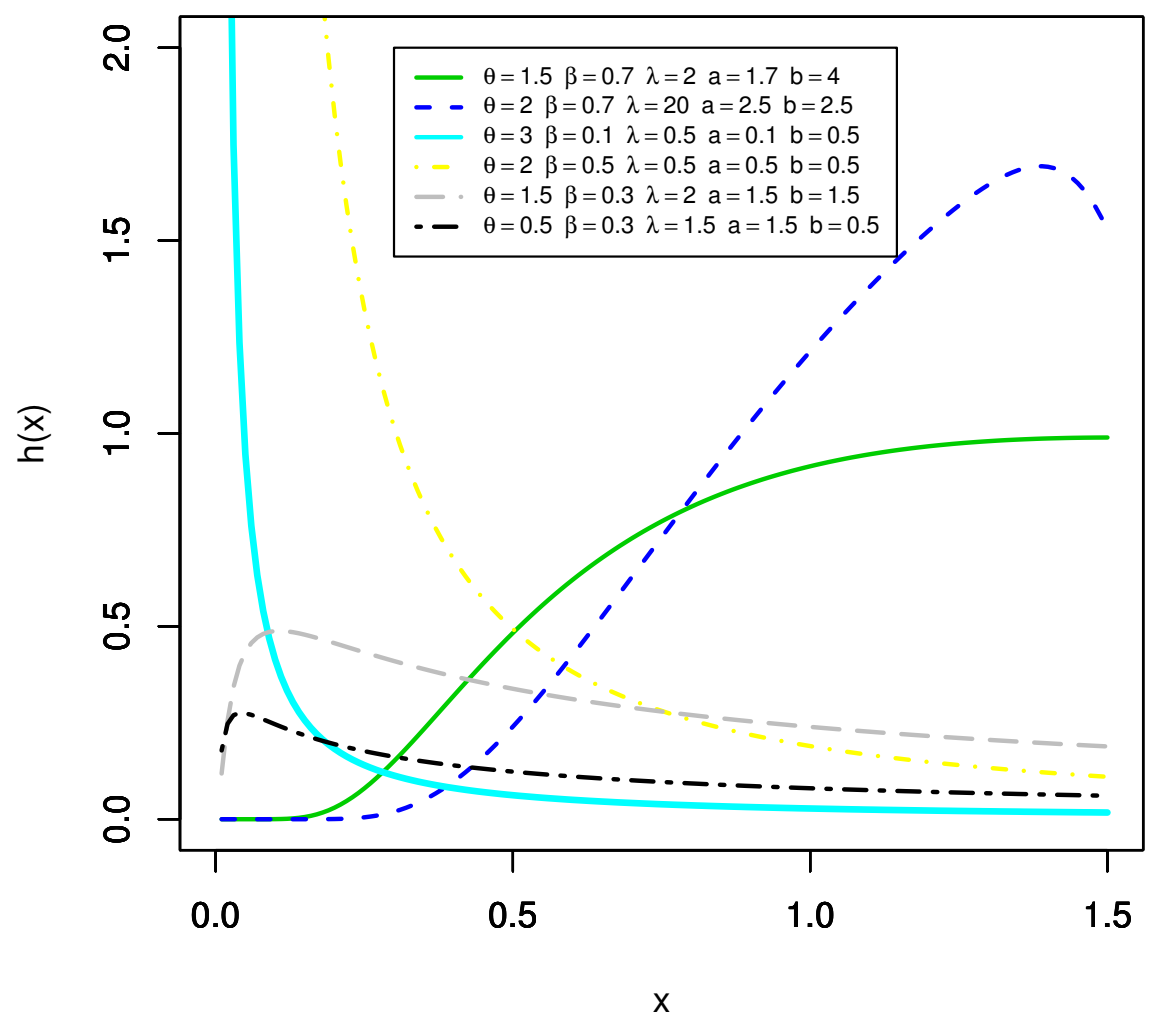

Figure 2: The hrf plots for the BExFr model.

Theorem 1. Suppose $X \sim \operatorname{BExFr}\left(a, b, \lambda_{1}, \beta_{1}, \theta_{1}\right)$ and $Y \sim \operatorname{BExFr}\left(a, b, \lambda_{2}, \beta_{2}, \theta_{2}\right)$. If $\beta_{1}<\beta_{2}$, $\theta_{1}>\theta_{2}$ and $\lambda_{1}<\lambda_{2}$ Then $X \underset{\text { st }}{\geqslant} Y$, for integer values of $\beta_{1}$ and $\beta_{2}$.

Proof. At first, we consider the following:

$$
I_{x}(a, b)=\frac{\int_{0}^{x} u^{a-1}(1-u)^{b-1} d u}{B(a, b)} .
$$

Next, note that the incomplete beta function $\operatorname{Beta}_{x}(a, b)$ is an increasing function of $x$ for fixed $a$ and $b$. For any real number $x \in(0, \infty), \beta_{1}<\beta_{2}, \theta_{1}>\theta_{2}$ and $\lambda_{1}<\lambda_{2}$, we have

$$
\left[1-e^{-\left(\frac{\theta_{1}}{x}\right)^{\beta_{1}}}\right]^{\lambda_{1}}>\left[1-e^{-\left(\frac{\theta_{2}}{x}\right)^{\beta_{2}}}\right]^{\lambda_{2}}
$$

This implies that $I\left[1-e^{-\left(\frac{\theta_{1}}{x}\right)^{\beta_{1}}}\right]^{\lambda_{1}(a, b) \leq I}\left[1-e^{-\left(\frac{\theta_{2}}{x}\right)^{\beta_{2}}}\right]^{\lambda_{2}(a, b) \text {. Equivalently, it implies that }}$ $P(X>x) \geq P(Y>x)$ and this completes the proof.

Note: For fractional choices of $\lambda_{1}, \lambda_{2}, \beta_{1}, \beta_{2}$, the reverse of the theorem will be observed.

Corollary 1. From Theorem 1, we conjecture the following:

- For increasing $\theta$, and $\beta$ and $\lambda$ decreasing, the hrf will exhibit DFR. 
- For decreasing $\theta$, and $\beta$ and $\lambda$ increasing, the hrf will exhibit IFR.

\subsection{Quantile function}

Let $Q_{a, b}(u)$ be the beta qf with parameters $a$ and $b$. The qf of the BExFr distribution, say $x=Q(u)$, is given by

$$
Q(u)=\theta\left\{-\ln \left[1-\left[1-Q_{a, b}(u)\right]^{1 / \lambda}\right]\right\}^{-1 / \beta}, 0<u<1 .
$$

This scheme is useful to generate BFr random variates because of the existence of fast generators for beta random variables in most statistical packages, i.e. if $V$ is a beta random variable with parameters $a$ and $b$, then

$$
X=\theta\left\{-\ln \left[1-(1-V)^{1 / \lambda}\right]\right\}^{-1 / \beta}
$$

follows the BExFr distribution.

\subsection{Moments}

Henceforth, let $Z$ be a random variable having the Fréchet distribution (1) with parameters $\theta$ and $\beta$. For $r<\beta$, the $r$ th ordinary and incomplete moments of $Z$ are, respectively, given by

$$
\mu_{r}^{\prime}=\theta^{r} \Gamma(1-r / \beta) \quad \text { and } \quad \varphi_{r}(t)=\theta^{r} \gamma\left(1-r / \beta,(\theta / t)^{\beta}\right),
$$

where $\gamma(s, t)=\int_{0}^{t} x^{s-1} e^{-x} d x$ is the lower incomplete gamma function.

Then, the $r$ th moment of $X$, say $\mu_{r}^{\prime}$, can be expressed as

$$
\mu_{r}^{\prime}=\theta^{r} \sum_{k=0}^{\infty} v_{k}(k+1)^{r / \beta} \Gamma(1-r / \beta) .
$$

Setting $r=1$ in (9), we have the mean of $X$.

Using the relation between the central and non-central moments, we obtain the $n$th central moment of $X$, say $\mu_{n}$, as follows

$$
\mu_{n}=\theta^{r} \sum_{r=0}^{n} \sum_{k=0}^{\infty}\left(\begin{array}{l}
n \\
r
\end{array}\right) v_{k}\left(-\mu_{1}^{\prime}\right)^{n-r}(k+1)^{r / \beta} \Gamma(1-r / \beta) .
$$

The skewness and kurtosis measures can be determined from the central moments using wellknown relationships.

\subsection{Moment generating function}

First, we provide the generating function of the Fréchet model as discussed by Afify et al. (2016). Setting $y=x^{-1}$, we can write the mgf of $Z$ as

$$
M(t ; \theta, \beta)=\beta \theta^{\beta} \int_{0}^{\infty} e^{\frac{t}{y}} y^{\beta-1} e^{-(\theta y)^{\beta}} d y .
$$

After expanding $e^{\frac{t}{y}}$, we can write

$$
\begin{aligned}
M(t ; \theta, \beta) & =\beta \theta^{\beta} \int_{0}^{\infty} \sum_{m=0}^{\infty} \frac{t^{m}}{m !} y^{\beta-m-1} e^{-(\theta y)^{\beta}} d y \\
& =\sum_{m=0}^{\infty} \frac{\theta^{m} t^{m}}{m !} \Gamma\left(\frac{\beta-m}{\beta}\right),
\end{aligned}
$$


where the gamma function is well-defined for any non-integer $\beta$.

Consider the Wright generalized hypergeometric function defined by

$$
{ }_{p} \Psi_{q}\left[\begin{array}{l}
\left(\gamma_{1}, A_{1}\right), \ldots,\left(\gamma_{p}, A_{p}\right) \\
\left(\beta_{1}, B_{1}\right), \ldots,\left(\beta_{q}, B_{q}\right)
\end{array} ; x\right]=\sum_{n=0}^{\infty} \frac{\prod_{j=1}^{p} \Gamma\left(\gamma_{j}+A_{j} n\right)}{\prod_{j=1}^{q} \Gamma\left(\beta_{j}+B_{j} n\right)} \frac{x^{n}}{n !} .
$$

Then, we can write $M(t ; \theta, \beta)$ as

$$
M(t ; \theta, \beta)={ }_{1} \Psi_{0}\left[\begin{array}{c}
\left(1,-\beta^{-1}\right) \\
-
\end{array} ; \theta t\right] .
$$

Combining expressions (8) and the last equation, we obtain the mgf of $X$, say $M(t)$, as

$$
M(t)=\sum_{k=0}^{\infty} v_{k 1} \Psi_{0}\left[\begin{array}{c}
\left(1,-\beta^{-1}\right) \\
-
\end{array} ; \theta(k+1)^{1 / \beta} t\right] .
$$

\subsection{Incomplete moments}

The $n$th incomplete moment, say $\vartheta_{n}(t)$ of the BExFr model is given by $\vartheta_{n}(t)=\int_{0}^{t} x^{n} f(x) d x$. From equation (8), we can write

$$
\vartheta_{n}(t)=\sum_{k=0}^{\infty} v_{k} \int_{0}^{t} x^{n} h_{k+1}(x) .
$$

Using the lower incomplete gamma function, we obtain (for $n<\beta$ )

$$
\vartheta_{n}(t)=\sum_{k=0}^{\infty} v_{k} \theta^{n}(k+1)^{n / \beta} \gamma\left(1-\frac{n}{\beta},(k+1)\left(\frac{\theta}{t}\right)^{\beta}\right) .
$$

The important application of the first incomplete moment is related to the Bonferroni and Lorenz curves. These curves are very useful in economics, reliability, demography, insurance and medicine.

Further, the amount of scatter in a population is evidently measured to some extent by the totality of deviations from the mean and median. The mean deviations about the mean and about the median of $X$ can be expressed as $\delta_{\mu}(X)=\int_{0}^{\infty}\left|X-\mu_{1}^{\prime}\right| f(x) d x=2 \mu_{1}^{\prime} F\left(\mu_{1}^{\prime}\right)-$ $2 \vartheta_{1}\left(\mu_{1}^{\prime}\right)$ and $\delta_{M}(X)=\int_{0}^{\infty}|X-M| f(x) d x=\mu_{1}^{\prime}-2 \vartheta_{1}(M)$, respectively, where $\mu_{1}^{\prime}=E(X)$ comes from (11), $F\left(\mu_{1}^{\prime}\right)$ is simply calculated from $(5), \vartheta_{1}\left(\mu_{1}^{\prime}\right)$ is the first incomplete moment and $M$ is the median of $X$.

\subsection{Mean residual life and mean inactivity time}

The MRL has many applications in biomedical sciences, life insurance, maintenance and product quality control, economics and social studies, demography and product technology (see Lai and Xie, 2006). Guess and Proschan (1988) gave an extensive coverage of possible applications of the mean residual life. The MRL (or the life expectancy at age $t$ ) represents the expected additional life length for a unit, which is alive at age $t$.

The MRL is given by

$$
m_{X}(t)=E(X-t \mid X>t), t>0 .
$$

Then, the MRL of $X$ can be obtained as

$$
m_{X}(t)=\left[1-\vartheta_{1}(t)\right] / R(t)-t
$$


where $\vartheta_{1}(t)$ is the first incomplete moment of $X$ and by setting $n=1$ in equation (10), we obtain

$$
\vartheta_{1}(t)=\sum_{k=0}^{\infty} v_{k} \theta(k+1)^{1 / \beta} \gamma\left(1-\frac{1}{\beta},(k+1)\left(\frac{\theta}{t}\right)^{\beta}\right) .
$$

By substituting (12) in equation (11), we obtain

$$
m_{X}(t)=\frac{\theta}{R(t)} \sum_{k=0}^{\infty} v_{k}(k+1)^{1 / \beta} \gamma\left(1-\frac{1}{\beta},(k+1)\left(\frac{\theta}{t}\right)^{\beta}\right)-t .
$$

The MIT represents the waiting time elapsed since the failure of an item on condition that this failure had occurred in $(0, t)$. The MIT of $X$ is defined (for $t>0$ ) by $M_{X}(t)=$ $E(t-X \mid X \leq t)$.

The MIT of $X$ is given by

$$
M_{X}(t)=t-\left[\varphi_{1}(t) / F(t)\right] .
$$

By inserting (12) in equation (13), we obtain the MIT of $X$ as

$$
M_{X}(t)=t-\frac{\theta}{F(t)} \sum_{k=0}^{\infty} v_{k}(k+1)^{1 / \beta} \gamma\left(1-\frac{1}{\beta},(k+1)\left(\frac{\theta}{t}\right)^{\beta}\right) .
$$

\subsection{Entropies}

The Rényi entropy of a random variable $X$ represents a measure of variation of the uncertainty. The Rényi entropy is defined by

$$
I_{q}(x)=\frac{1}{1-q} \log \int_{-\infty}^{\infty} f^{q}(x) d x, q>0 \text { and } q \neq 1 .
$$

From equation (5), we can write

$$
\begin{aligned}
f^{q}(x)= & \left(\frac{\lambda \beta \theta^{\beta}}{B(a, b)}\right)^{q} x^{-q(\beta+1)} e^{-q\left(\frac{\theta}{x}\right)^{\beta}}\left[1-e^{-\left(\frac{\theta}{x}\right)^{\beta}}\right]^{q(\lambda b-1)} \\
& \times\left\{1-\left[1-e^{-\left(\frac{\theta}{x}\right)^{\beta}}\right]^{\lambda}\right\}^{q(a-1)} .
\end{aligned}
$$

Applying the power series (6) to the last equation and after some simplifications, we can write

$$
f^{q}(x)=\beta^{q} \theta^{q \beta} \sum_{k=0}^{\infty} \omega_{k} x^{-q(\beta+1)} e^{-(k+q)\left(\frac{\theta}{x}\right)^{\beta}},
$$

where

$$
\omega_{k}=\sum_{j=0}^{\infty} \frac{(-1)^{j+k} \lambda^{q} \Gamma(q(\lambda b-1)+1) \Gamma(\lambda(b q+j)-q+1)}{j ! k ![B(a, b)]^{q} \Gamma(q(\lambda b-1)-j+1) \Gamma(\lambda(b q+j)-q-k+1)} .
$$

Then, the Rényi entropy of $X$ is given by

$$
I_{q}(x)=\frac{1}{1-q} \log [\beta^{q} \theta^{q \beta} \sum_{k=0}^{\infty} \omega_{k} \underbrace{\int_{0}^{\infty} x^{-q(\beta+1)} e^{-(k+q)\left(\frac{\theta}{x}\right)^{\beta}} d x}_{I}] .
$$

Then,

$$
I=\frac{\theta^{1-q(\beta+1)}}{\beta}(k+q)^{-s / \beta} \Gamma\left(\frac{s}{\beta}\right),
$$


where $s=q(\beta+1)-1$.

Now, we can write the Rényi entropy of $X$ as

$$
I_{q}(x)=\frac{1}{1-q} \log \left\{\sum_{k=0}^{\infty} \omega_{k}\left(\frac{\beta}{\theta}\right)^{q-1}(k+q)^{-s / \beta} \Gamma\left(\frac{s}{\beta}\right)\right\} .
$$

The q-entropy, say $H_{q}(x)$, is defined by

$$
H_{q}(x)=\frac{1}{q-1} \log \left[1-I_{q}(x)\right],
$$

where

$$
I_{q}(x)=\int_{\Re} f^{q}(x) d x, q>0 \text { and } q \neq 1 .
$$

From equation (14), we obtain

$$
H_{q}(f)=\frac{1}{q-1} \log \left\{1-\sum_{k=0}^{\infty} \omega_{k}\left(\frac{\beta}{\theta}\right)^{q-1}(k+q)^{-s / \beta} \Gamma\left(\frac{s}{\beta}\right)\right\} .
$$

The Shannon entropy, say $E_{s h}$, of a random variable $X$ is defined by

$$
E_{s h}=E\{-[\log f(x)]\} .
$$

It is a special case of the Rényi entropy when $q \uparrow 1$. So, based on equation (8), we can write

$$
E_{s h}=-\left\{\log \left[\sum_{k=0}^{\infty} v_{k} E\left(Y_{k+1}\right)\right]\right\},
$$

where $Y_{k+1} \sim \operatorname{Fr}\left(\theta(k+1)^{1 / \beta}, \beta\right)$. But $E\left(Y_{k+1}\right)=\theta(k+1)^{1 / \beta} \Gamma(1-1 / \beta)$, so the $E_{\text {sh }}$ of $X$ is given by

$$
E_{s h}=-\left\{\log \left[\sum_{k=0}^{\infty} v_{k} \theta(k+1)^{1 / \beta} \Gamma\left(1-\frac{1}{\beta}\right)\right]\right\} .
$$

\section{Order statistics}

In this section we consider the expression for the general $r$-th order statistic and the large sample distribution of the sample minimum and the sample maximum when a random sample of size $n$ are drawn from the $\operatorname{BExFr}(a, b, \lambda, \beta, \theta)$ distribution. The density function of the $r$ th order statistic, $X_{r: n}$, for a random sample of size $n$ drawn from (5), is given by

$$
f_{X_{r: n}}(x)=\frac{1}{B(r, n-r+1)}(F(x))^{r-1}(1-F(x))^{n-r} f(x) .
$$

Then the $r$ th order statistic of $X$ is given by

$$
\begin{aligned}
f_{X_{r: n}}(x)= & \frac{1}{B(r, n-r+1)} f(x) \sum_{j=0}^{n-r}(-1)^{j}\left(\begin{array}{c}
n-r \\
j
\end{array}\right) I(0<x<1) \\
& \times\left(\frac{B\left(1-\left(1-e^{\left.\left.-\left(\frac{\theta}{x}\right)^{\beta}\right)^{\lambda} ; a, b\right)}\right.\right.}{B(a, b)}\right)
\end{aligned}
$$

Using the series expression for the incomplete beta function: 


$$
I_{x}(a, b,)=\frac{B(x, a, b)}{B(a, b)}=\sum_{k=a}^{a+b-1} x^{k}(1-x)^{a+b-1-k},
$$

the pdf of $X_{r: n}$ can be written as

$$
\begin{aligned}
f_{r: n}(x) & =\frac{f(x)}{B(r, n-r+1)} \sum_{j=0}^{n-r} \sum_{k=a}^{a+b-1}(-1)^{j}\left(\begin{array}{c}
n-r \\
j
\end{array}\right) \\
& \times\left\{\sum_{k=a}^{a+b-1}\left[1-\left(1-e^{-\left(\frac{\theta}{x}\right)^{\beta}}\right)^{\lambda}\right]^{k}\left(1-e^{-\left(\frac{\theta}{x}\right)^{\beta}}\right)^{\lambda(a+b-1-k)}\right\}^{r-1+j} \\
& =\frac{f(x)}{B(r, n-r+1)} \sum_{j=0}^{n-r} \sum_{k_{1}=a}^{a+b-1} \ldots \sum_{k_{r-1+j}=a}^{a+b-1}(-1)^{j+s_{k}}\left(\begin{array}{c}
n-r \\
j
\end{array}\right) \\
& \times \frac{\operatorname{Beta}\left(s_{k}+a,(r-1+j)(a+2 b-1)-s_{k}\right)}{\operatorname{Beta}(a, b)} p_{k} \\
& \left.\times f\left(x \mid s_{k}+a,(r-1+j)(a+2 b-1)-s_{k}\right), \lambda, \beta, \theta\right), \\
\text { where } s_{k}=\sum_{i=1}^{r-1+j} & k_{i} \text { and } p_{k}=\prod_{i=1}^{r-1+j}\left(\begin{array}{c}
a+b-1 \\
k_{i}
\end{array}\right) .
\end{aligned}
$$

Also, $\left.f\left(x \mid s_{k}+a,(r-1+j)(a+2 b-1)-s_{k}\right), \lambda, \beta, \theta\right)$ is the density of a BExFr distribution with parameters $\left.s_{k}+a,(r-1+j)(a+2 b-1)-s_{k}\right), \lambda, \beta, \theta$ respectively.

From (15), it is interesting to note that the pdf of the $r$ th order statistic $X_{r: n}$ can be expressed as an finite sums of the BExFr pdf 's. However, if $a$ and $b$ are not integers, then the sums will terminate at $\infty$. Note that, using moments expression, one can easily get an expression for the general $m$ th order moment for the order statistics.

\section{Characterizations}

This section deals with various characterizations of BExFr distribution. These characterizations are based on: $(i)$ a simple relationship between two truncated moments; $(i i)$ the hazard function. It should be mentioned that for characterization $(i)$ the cdf need no have a closed form. We believe, due to the nature of the cdf of BExFr, there may not be other possible characterizations than the ones presented in this section.

\subsection{Characterizations based on two truncated moments}

In this subsection we present characterizations of BExFr distribution in terms of a simple relationship between two truncated moments. Our first characterization employs a theorem due to Glänzel (1987), see Theorem 1 below. Note that the result holds also when the interval $H$ is not closed. It should also be mentioned that this characterization is stable in the sense of weak convergence.

Theorem 2. Let $(\Omega, \mathcal{F}, \mathbf{P})$ be a given probability space and let $H=[d, e]$ be an interval for some $d<e \quad(d=-\infty, e=\infty$ might as well be allowed). Let $X: \Omega \rightarrow H$ be a continuous random variable with the distribution function $F$ and let $g$ and $h$ be two real functions defined on $H$ such that

$$
\mathbf{E}[g(X) \mid X \geq x]=\mathbf{E}[h(X) \mid X \geq x] \eta(x), \quad x \in H,
$$

is defined with some real function $\eta$. Assume that $g, h \in C^{1}(H), \eta \in C^{2}(H)$ and $F$ is twice continuously differentiable and strictly monotone function on the set $H$. Finally, assume that 
the equation $h \eta=g$ has no real solution in the interior of $H$. Then $F$ is uniquely determined by the functions $g, h$ and $\eta$, particularly

$$
F(x)=\int_{a}^{x} C\left|\frac{\eta^{\prime}(u)}{\eta(u) h(u)-g(u)}\right| \exp (-s(u)) d u,
$$

where the function $s$ is a solution of the differential equation $s^{\prime}=\frac{\eta^{\prime} h}{\eta h-g}$ and $C$ is the normalization constant, such that $\int_{H} d F=1$.

Proposition 1. Let $X: \Omega \rightarrow(0, \infty)$ be a continuous random variable and let

$$
h(x)=\left[1-\left(1-e^{-\left(\frac{\theta}{x}\right)^{\beta}}\right)^{\lambda}\right]^{1-a} \text { and } g(x)=h(x)\left(1-e^{-\left(\frac{\theta}{x}\right)^{\beta}}\right) \text { for } x>0 .
$$

The random variable $X$ belongs to BExFr family (5) if and only if the function $\eta$ defined in Theorem 2 has the form

$$
\eta(x)=\frac{\lambda b}{\lambda b+1}\left(1-e^{-\left(\frac{\theta}{x}\right)^{\beta}}\right), \quad x>0 .
$$

Proof. Let $X$ be a random variable with density (5), then

$$
(1-F(x)) E[h(x) \mid X \geq x]=\frac{1}{b B(a, b)}\left(1-e^{-\left(\frac{\theta}{x}\right)^{\beta}}\right)^{\lambda b}, x>0
$$

and

$$
(1-F(x)) E[g(x) \mid X \geq x]=\frac{\lambda}{(\lambda b+1) B(a, b)}\left(1-e^{-\left(\frac{\theta}{x}\right)^{\beta}}\right)^{\lambda b+1}, x>0,
$$

and finally

$$
\eta(x) h(x)-g(x)=h(x) \frac{1}{b B(a, b)}\left(1-e^{-\left(\frac{\theta}{x}\right)^{\beta}}\right)\left\{-\frac{1}{\lambda b+1}\right\}<0 \text { for } x>0 .
$$

Conversely, if $\eta$ is given as above, then

$$
s^{\prime}(x)=\frac{\eta^{\prime}(x) h(x)}{\eta(x) h(x)-g(x)}=\frac{\lambda b \beta x^{-(\beta+1)} e^{-\left(\frac{\theta}{x}\right)^{\beta}}}{1-e^{-\left(\frac{\theta}{x}\right)^{\beta}}}, \quad x>0
$$

and hence

$$
s(x)=-\ln \left\{\left(1-e^{-\left(\frac{\theta}{x}\right)^{\beta}}\right)^{\lambda b}\right\}, \quad x>0 .
$$

Now, in view of Theorem 2, $X$ has density (5).

Corollary 2. Let $X: \Omega \rightarrow(0, \infty)$ be a continuous random variable and let $h(x)$ be as in Proposition 1. The pdf of $X$ is (5) if and only if there exist functions $g$ and $\eta$ defined in Theorem 2 satisfying the differential equation

$$
\frac{\eta^{\prime}(x) h(x)}{\eta(x) h(x)-g(x)}=\frac{\lambda b \beta x^{-(\beta+1)} e^{-\left(\frac{\theta}{x}\right)^{\beta}}}{1-e^{-\left(\frac{\theta}{x}\right)^{\beta}}}, \quad x>0 .
$$

The general solution of the differential equation in Corollary 2 is 


$$
\eta(x)=\left[1-e^{-\left(\frac{\theta}{x}\right)^{\beta}}\right]^{-\lambda b}\left[-\int^{\lambda b \beta e^{-\left(\frac{\theta}{x}\right)^{\beta}}\left[1-e^{-\left(\frac{\theta}{x}\right)^{\beta}}\right]^{\lambda b-1}} g(x) d x+D\right]
$$

where $D$ is a constant. Note that a set of functions satisfying the differential equation (16) is given in Proposition 1 with $D=0$. Clearly, there are other triplets $(h, g, \eta)$ satisfying the conditions of Theorem 2.

\subsection{Characterization based on hazard function}

It is known that the hazard function, $h_{F}$, of a twice differentiable distribution function, $F$, satisfies the first order differential equation

$$
\frac{f^{\prime}(x)}{f(x)}=\frac{h_{F}^{\prime}(x)}{h_{F}(x)}-h_{F}(x)
$$

For many univariate continuous distributions, this is the only characterization available in terms of the hazard function. The following characterization establish a non-trivial characterization for BExFr distribution in terms of the hazard function when $a=1$, which is not of the trivial form given in (17).

Proposition 2. Let $X: \Omega \rightarrow(0, \infty)$ be a continuous random variable. Then for $a=1$, the pdf of $X$ is (5) if and only if its hazard function $h_{F}(x)$ satisfies the differential equation

$$
\begin{aligned}
h_{F}^{\prime}(x)+(\beta+1) x^{-1} h_{F}(x) & =\lambda b \beta^{2} \theta^{2 \beta} x^{-2(\beta+1)} e^{-\left(\frac{\theta}{x}\right)^{\beta}}\left(1-e^{-\left(\frac{\theta}{x}\right)^{\beta}}\right)^{-2} \\
& =\lambda b \beta \theta^{\beta} x^{-(\beta+1)} \frac{d}{d x}\left\{\left(1-e^{-\left(\frac{\theta}{x}\right)^{\beta}}\right)^{-1}\right\} .
\end{aligned}
$$

Proof. If $X$ has pdf (5), then clearly (18) holds. Now, if (18) holds, then

$$
\frac{d}{d x}\left\{x^{\beta+1} h_{F}(x)\right\}=\lambda b \beta \theta^{\beta} \frac{d}{d x}\left\{\left(1-e^{-\left(\frac{\theta}{x}\right)^{\beta}}\right)^{-1}\right\}
$$

or, equivalently,

$$
h_{F}(x)=\frac{\lambda b \beta \theta^{\beta} x^{-(\beta+1)} e^{-\left(\frac{\theta}{x}\right)^{\beta}}}{1-e^{-\left(\frac{\theta}{x}\right)^{\beta}}} .
$$

Integrating the above equation from 0 to $x$, we obtain

$$
1-F(x)=\left(1-e^{-\left(\frac{\theta}{x}\right)^{\beta}}\right)^{\lambda b}, x \geq 0 .
$$

\section{Maximum likelihood estimation}

In this section, we consider the estimation of the parameters of the BExFr model by the maximum likelihood. Consider the random sample $X_{1}, \ldots, X_{n}$ of size $n$ from this distribution. 
The log-likelihood function for the parameter vector $\varphi=(a, b, \lambda, \beta, \theta)^{\top}$, say $\ell(\varphi)$, is given by

$$
\begin{aligned}
\ell(\varphi)= & n[\log \lambda+\log \beta+\beta \log \theta-\log B(a, b)]-(\beta+1) \sum_{i=1}^{n} \log \left(x_{i}\right) \\
& -\sum_{i=1}^{n}\left(\frac{\theta}{x_{i}}\right)^{\beta}+(\lambda b-1) \sum_{i=1}^{n} \log \left(s_{i}\right)+(a-1) \sum_{i=1}^{n} \log \left(1-s_{i}^{\lambda}\right),
\end{aligned}
$$

where $s_{i}=1-e^{-\left(\frac{\theta}{x_{i}}\right)^{\beta}}$.

This equation can be maximized either directly by using the $\mathrm{R}$ (optim function), MATH-CAD program, SAS (PROC NLMIXED) or by solving the nonlinear equations obtained by differentiating the log-likelihood. Therefore, the score vector is $\mathbf{U}(\varphi)=\frac{\partial \ell}{\partial \varphi}=\left(\frac{\partial \ell}{\partial a}, \frac{\partial \ell}{\partial b}, \frac{\partial \ell}{\partial \lambda}, \frac{\partial \ell}{\partial \beta}, \frac{\partial \ell}{\partial \theta}\right)^{\top}$.

$$
\begin{gathered}
\frac{\partial \ell}{\partial a}=n[\psi(a+b)-\psi(a)]+\sum_{i=1}^{n} \log \left(1-s_{i}^{\lambda}\right), \\
\frac{\partial \ell}{\partial b}=n[\psi(a+b)-\psi(b)]+\lambda \sum_{i=1}^{n} \log \left(s_{i}\right), \\
\frac{\partial \ell}{\partial \lambda}=\frac{n}{\lambda}+b \sum_{i=1}^{n} \log \left(s_{i}\right)-(a-1) \sum_{i=1}^{n} \frac{s_{i}^{\lambda} \log \left(s_{i}\right)}{1-s_{i}^{\lambda}} \\
\frac{\partial \ell}{\partial \beta}=\frac{n}{\beta}+n \log \theta-\sum_{i=1}^{n} \log \left(x_{i}\right)-\sum_{i=1}^{n}\left(\frac{\theta}{x_{i}}\right)^{\beta} \log \left(\frac{\theta}{x_{i}}\right) \\
+(\lambda b-1) \sum_{i=1}^{n} \frac{z_{i}}{s_{i}}-\lambda(a-1) \sum_{i=1}^{n} \frac{z_{i} s_{i}^{\lambda-1}}{1-s_{i}^{\lambda}}
\end{gathered}
$$

and

$$
\begin{aligned}
\frac{\partial \ell}{\partial \theta}= & \frac{n \beta}{\theta}-\frac{\beta}{\theta} \sum_{i=1}^{n}\left(\frac{\theta}{x_{i}}\right)^{\beta}-\frac{\beta \lambda(a-1)}{\theta} \sum_{i=1}^{n} \frac{\left(\frac{\theta}{x_{i}}\right)^{\beta} s_{i}^{\lambda-1}}{1-s_{i}^{\lambda}} \\
& +\frac{\beta(\lambda b-1)}{\theta} \sum_{i=1}^{n} \frac{1}{s_{i}}\left(\frac{\theta}{x_{i}}\right)^{\beta} e^{-\left(\frac{\theta}{x_{i}}\right)^{\beta}}
\end{aligned}
$$

where $z_{i}=\left(\frac{\theta}{x_{i}}\right)^{\beta} e^{-\left(\frac{\theta}{x_{i}}\right)^{\beta}} \log \left(\frac{\theta}{x_{i}}\right)$ and $\psi($.$) is the digamma function which is the derivative$ of $\log \Gamma($.$) , where \Gamma($.$) is the gamma function.$

We can obtain the estimates of the unknown parameters by setting the score vector to zero, $\mathbf{U}(\widehat{\varphi})=0$. Solving these equations simultaneously yields the MLEs $\widehat{\varphi}=(\widehat{a}, \widehat{b}, \widehat{\lambda}, \widehat{\beta}, \widehat{\theta})^{\top}$ of $\varphi=(a, b, \lambda, \beta, \theta)^{\top}$. These equations cannot be solved analytically and statistical software can be used to solve them numerically by means of iterative techniques such as the NewtonRaphson algorithm. For the new distribution all the second-order derivatives exist.

For interval estimation of the model parameters, we require the $5 \times 5$ observed information matrix $J(\varphi)=\left\{J_{r s}\right\} \quad$ (for $\left.r, s=a, b, \lambda, \beta, \theta\right)$. Under standard regularity conditions, the multivariate normal $N_{5}\left(0, J(\widehat{\varphi})^{-1}\right)$ distribution can be used to construct approximate confidence intervals for the model parameters. Here, $J(\widehat{\varphi})$ is the total observed information matrix evaluated at $\widehat{\varphi}$. Based on this multivariate normal approximation, the approximate $100(1-\phi) \%$ confidence intervals for $a, b, \lambda, \beta$ and $\theta$ can be determined by the usual way. 


\section{Simulation}

In this section, we consider the maximum likelihood estimation of parameters for the two models derived in the preceding sections. The maximum likelihood estimators can be obtained by direct maximization of the likelihood functions given earlier. Here, we maximized the loglikelihood function using SAS PROC NLMIXED. For each maximization, the SAS PROC NLMIXED function was executed for a wide range of initial values, and the maximum likelihood estimates were determined as the ones that corresponds to the largest of the maxima.

To illustrate the feasibility of the suggested estimation strategy, a small simulation study was undertaken. The simulation study was carried out for one representative set of parameters $(\lambda, \beta, \theta, a, b)=(1.6,2.3,1.2,1.8,0.9)$ and the process was repeated 30000 times. Three different sample sizes $n=50,100$ and 200 were considered. The bias (actual-estimate) and the standard deviation of the parameter estimates for the maximum likelihood estimates were determined from this simulation study and are presented in Table 2 .

Table 2. Bias and standard deviation of the parameter estimates.

\begin{tabular}{|c|c|c|c|}
\hline Parameter & Sample size $(n=50)$ & Sample size $(n=100)$ & Sample size $(n=200)$ \\
\hline$\lambda$ & $0.1108(0.5382)$ & $0.0614(0.2345)$ & $0.0437(0.1139)$ \\
\hline$\beta$ & $0.1678(0.4628)$ & $-0.1321(0.1894)$ & $0.0672(0.0933)$ \\
\hline$\theta$ & $0.0268(0.4321)$ & $0.1483(0.2467)$ & $0.0946(0.1264)$ \\
\hline$a$ & $0.0825(0.0667)$ & $0.0779(0.0627)$ & $0.0621(0.0358)$ \\
\hline$b$ & $0.127(0.2368)$ & $0.0651(0.0789)$ & $0.0317(0.0223)$ \\
\hline
\end{tabular}

The figures in Table 2 indicate that the estimates are quite stable and, more important, are close to the true values for the these sample sizes. Furthermore, as the sample size increases, the SEs decreases as expected.

\section{Applications}

In this section, we provide two applications to two real data sets to prove the importance and flexibility of the BExFr distribution. The first real data set represents the survival times, in weeks, of 33 patients suffering from acute Myelogeneous Leukaemia. These data have been analyzed by Feigl and Zelen (1965). The data are: 65, 156, 100, 134, 16, 108, 121, 4, 39, 143, $56,26,22,1,1,5,65,56,65,17,7,16,22,3,4,2,3,8,4,3,30,4,43$. The second data set, strength data, which were originally reported by Badar and Priest (1982) and it represents the strength measured in GPA for single carbon fibers and impregnated 1000-carbon fiber tows. Single fibers were tested under tension at gauge lengths of $10 \mathrm{~mm}$ with sample size $(\mathrm{n}=63)$. This data set consists of observations: 1.901, 2.132, 2.203, 2.228, 2.257, 2.350, 2.361, 2.396, $2.397,2.445,2.454,2.474,2.518,2.522,2.525,2.532,2.575,2.614,2.616,2.618,2.624,2.659$, $2.675,2.738,2.740,2.856,2.917,2.928,2.937,2.937,2.977,2.996,3.030,3.125,3.139,3.145$, $3.220,3.223,3.235,3.243,3.264,3.272$, 3.294, 3.332, 3.346, 3.377, 3.408, 3.435, 3.493, 3.501, $3.537,3.554,3.562,3.628,3.852,3.871,3.886,3.971,4.024,4.027,4.225,4.395,5.020$. These data have been used by Afify et al. (2015b) and Afify et al. (2015c) to fit the exponentiated transmuted generalized Rayleigh and transmuted Weibull Lomax distributions, respectively.

We shall compare the fit of the proposed BExFr distribution (and its sub-models namely: $\mathrm{BFr}, \mathrm{EFr}$ and Fr distributions) with several other competitive models namely: the generalized inverse gamma (Mead, 2015), McDonald Lomax (McL) (Lemonte and Cordeiro, 2013), gamma Lomax (GL) ( Cordeiro et al., 2015) and Zografos-Balakrishnan log-logistic (ZBLL) (Zografos and Balakrishnan, 2009 ) models with corresponding densities (for $x>0$ ):

GIG: $f(x ; \theta, \beta, a, b, \lambda)=\frac{b \theta^{a b}}{\Gamma_{\lambda}(a, \beta)} x^{-(a b+1)}\left[\left(\frac{\theta}{x}\right)^{b}+\beta\right]^{-\lambda} \exp \left[-\left(\frac{\theta}{x}\right)^{b}\right] ;$
McL: $f(x ; \theta, \beta, a, b, \lambda)=\frac{\theta \lambda}{\beta B\left(\frac{a}{\lambda}, b\right)}\left(1+\frac{x}{\beta}\right)^{-(\theta+1)}\left[1-\left(1+\frac{x}{\beta}\right)^{-\theta}\right]^{a-1}$ 
$\times\left\{1-\left[1-\left(1+\frac{x}{\beta}\right)^{-\theta}\right]^{\lambda}\right\}^{b-1} ;$

GL: $f(x ; \theta, \beta, a)=\frac{\theta \beta^{\theta}}{\Gamma(a) \Gamma(\beta+x)^{\theta+1}}\left[-\theta \ln \left[\left(\frac{\beta}{\beta+x}\right)\right]\right]^{a-1} ;$

ZBLL: $f(x ; \theta, \beta, a)=\frac{\beta \theta^{-\beta}}{\Gamma(a)} x^{\beta-1}\left[1+\left(\frac{x}{\theta}\right)^{\beta}\right]^{-2}\left\{\ln \left[1+\left(\frac{x}{\theta}\right)^{\beta}\right]\right\}^{a-1}$;

where the parameters of the above densities are all positive real numbers, $\Gamma(a)$ is the gamma function and $\Gamma_{\lambda}(a, \beta)$ is the generalized gamma function (Kobayashi, 1991) defined by

$$
\Gamma_{\lambda}(a, \beta)=\int_{0}^{\infty} y^{a-1}(\beta+y)^{-\lambda} \exp (-y) d y .
$$

In order to compare the models, we consider some goodness-of-fit measures including $-2 \widehat{\ell}$, where $\widehat{\ell}$ is the maximized loglikelihood, Anderson-Darling $\left(A^{*}\right)$ and Cramér-von Mises $\left(W^{*}\right)$ statistics (full details can be found in Chen and Balakrishnan, 1995). In general, the model with minimum values for these statistics could be chosen as the best model to fit the data.

Tables 4 and 5 list the MLEs of the model parameters, their corresponding standard errors (given in parentheses) and the values of these statistics $\left(-2 \widehat{\ell}, A^{*}\right.$ and $\left.W^{*}\right)$ for the fitted models to both data sets.

Tables 3 and 4 compare the BExFr model with the BFr, EFr, Fr, GIG, McL, ZBLL and GL distributions. It is noted, from Tables 4 and 5 , that the BExFr distribution gives the lowest values for the $-2 \widehat{\ell}, A^{*}$ and $W^{*}$ statistics among all fitted models. Thus, the BExFr distribution could be chosen as the best models. These results are obtained using the MAT-HCAD PROGRAM.

Table 3. MLEs, their corresponding standard errors and the statistics $-2 \widehat{\ell}, W^{*}$ and $A^{*}$ for the first data set.

\begin{tabular}{|c|c|c|c|c|c|c|c|c|}
\hline \multirow[t]{2}{*}{ Model } & \multicolumn{5}{|c|}{ Estimates } & \multirow[t]{2}{*}{$-2 \widehat{\ell}$} & \multirow[t]{2}{*}{$W^{*}$} & \multirow[t]{2}{*}{$A^{*}$} \\
\hline & $\widehat{\theta}$ & $\widehat{\beta}$ & $\widehat{a}$ & $\widehat{b}$ & $\bar{\lambda}$ & & & \\
\hline $\mathrm{BExFr}$ & $\begin{array}{c}29.5877 \\
(201.769)\end{array}$ & $\begin{array}{c}0.1107 \\
(0.146)\end{array}$ & $\begin{array}{c}21.0415 \\
(64.505)\end{array}$ & $\begin{array}{c}19.7308 \\
(62.632)\end{array}$ & $\begin{array}{l}1.7253 \\
(4.578)\end{array}$ & 308.117 & 0.11211 & 0.70495 \\
\hline $\mathrm{BFr}$ & $\begin{array}{c}6.89494 \\
(60.760)\end{array}$ & $\begin{array}{l}0.13852 \\
(0.138)\end{array}$ & $\begin{array}{c}16.8911 \\
(43.704)\end{array}$ & $\begin{array}{l}24.04154 \\
(47.769)\end{array}$ & & 308.213 & 0.11407 & 0.71477 \\
\hline $\mathrm{EFr}$ & $\begin{array}{c}13.3423 \\
(14.377)\end{array}$ & $\begin{array}{l}0.5732 \\
(0.206)\end{array}$ & & & $\begin{array}{c}1.4581 \\
(1.007)\end{array}$ & 310.894 & 0.15728 & 0.95136 \\
\hline Fr & $\begin{array}{l}7.8651 \\
(2.091)\end{array}$ & $\begin{array}{c}0.6944 \\
(0.091)\end{array}$ & & & & 311.997 & 0.17306 & 1.0475 \\
\hline GIG & $\begin{array}{c}3.1481 \\
(45.860)\end{array}$ & $\begin{array}{c}0.89959 \\
(4.01682)\end{array}$ & $\begin{array}{c}8.42866 \\
(10.3956)\end{array}$ & $\begin{array}{l}0.29097 \\
(0.033)\end{array}$ & $\begin{array}{c}18.95355 \\
(17.1406)\end{array}$ & 308.233 & 0.11333 & 0.7127 \\
\hline McL & $\begin{array}{l}0.57665 \\
(1.067)\end{array}$ & $\begin{array}{c}8.46832 \\
(28.781)\end{array}$ & $\begin{array}{l}1.62102 \\
(2.762)\end{array}$ & $\begin{array}{l}1.72961 \\
(2.593)\end{array}$ & $\begin{array}{l}0.46481 \\
(3.251)\end{array}$ & 310.958 & 0.13714 & 0.84928 \\
\hline ZBLL & $\begin{array}{l}7.45579 \\
(0.248)\end{array}$ & $\begin{array}{l}1.01264 \\
(0.138)\end{array}$ & $\begin{array}{l}1.4687 \\
(0.179)\end{array}$ & & & 318.823 & 0.156487 & 0.95522 \\
\hline GL & $\begin{array}{l}1.1115 \\
(0.271)\end{array}$ & $\begin{array}{l}3.52517 \\
(6.722)\end{array}$ & $\begin{array}{c}2.1469 \\
(1.6968)\end{array}$ & & & 311.037 & 0.1419 & 0.86637 \\
\hline
\end{tabular}

Figures 3 and 4 display the estimated pdf's and cdf's of the BExFr distribution for the first real data set, where figures 5 and 6 represent the same for the second data set respectively. It is shown from these figures that the BExFr provides a close fit to these data sets. 


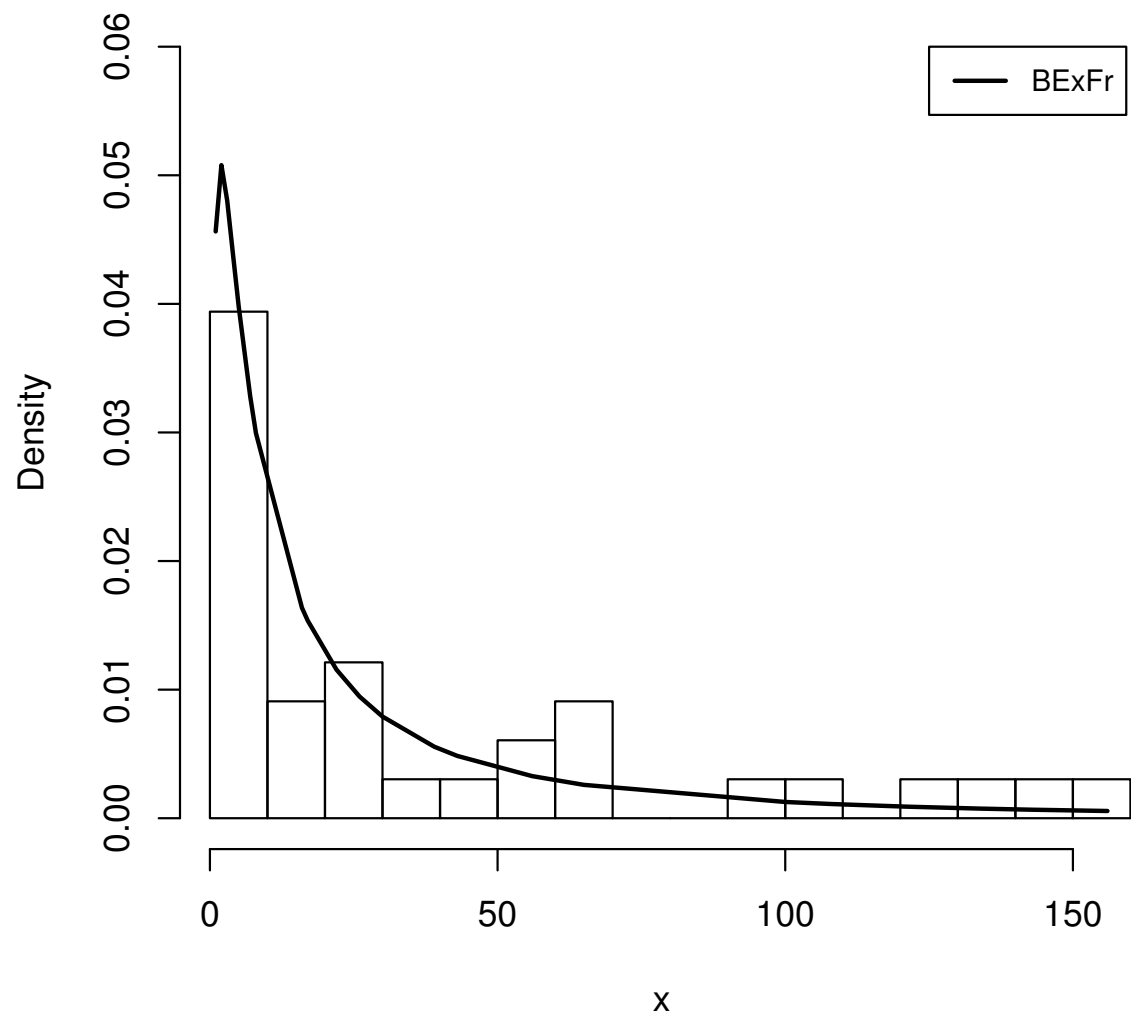

Figure 3: The fitted BExFr density for the first data set

Table 4. MLEs, their corresponding standard errors and the statistics $-2 \widehat{\ell}, W^{*}$ and $A^{*}$ the second data set

\begin{tabular}{|c|c|c|c|c|c|c|c|c|}
\hline \multirow[t]{2}{*}{ Model } & \multicolumn{5}{|c|}{ Estimates } & \multirow[t]{2}{*}{$\widehat{-2 \widehat{\ell}}$} & \multirow[t]{2}{*}{$W^{*}$} & \multirow[t]{2}{*}{$A^{*}$} \\
\hline & $\widehat{\theta}$ & $\widehat{\beta}$ & $\widehat{a}$ & $\widehat{b}$ & $\widehat{\lambda}$ & & & \\
\hline $\mathrm{BExFr}$ & $\begin{array}{l}12.6463 \\
(75.689)\end{array}$ & $\begin{array}{c}0.4852 \\
(0.978)\end{array}$ & $\begin{array}{c}25.7682 \\
(71.831)\end{array}$ & $\begin{array}{c}14.2299 \\
(67.197)\end{array}$ & $\begin{array}{c}7.2493 \\
(28.051)\end{array}$ & 112.704 & 0.05574 & 0.31204 \\
\hline $\mathrm{BFr}$ & $\begin{array}{l}25.03468 \\
(21.8041)\end{array}$ & $\begin{array}{l}0.55403 \\
(0.255)\end{array}$ & $\begin{array}{l}7.91533 \\
(6.395)\end{array}$ & $\begin{array}{l}185.80664 \\
(155.015)\end{array}$ & & 113.065 & 0.0558 & 0.32555 \\
\hline EFr & $\begin{array}{l}4.2957 \\
(1.613)\end{array}$ & $\begin{array}{c}2.3636 \\
(1.028)\end{array}$ & & & $\begin{array}{l}7.0322 \\
(8.513)\end{array}$ & 112.701 & 0.05895 & 0.31988 \\
\hline Fr & $\begin{array}{l}2.7214 \\
(0.067)\end{array}$ & $\begin{array}{c}5.4338 \\
(0.508)\end{array}$ & & & & 117.804 & 0.12884 & 0.69597 \\
\hline GIG & $\begin{array}{c}1.7724 \\
(0.5801)\end{array}$ & $\begin{array}{c}0.26685 \\
(1.18644)\end{array}$ & $\begin{array}{l}3.12731 \\
(3.3986)\end{array}$ & $\begin{array}{l}3.72201 \\
(2.082)\end{array}$ & $\begin{array}{c}8.12837 \\
(6.41594)\end{array}$ & 113.065 & 0.06669 & 0.3536 \\
\hline McL & $\begin{array}{l}1.89877 \\
(14.485)\end{array}$ & $\begin{array}{l}3.68277 \\
(10.640)\end{array}$ & $\begin{array}{c}37.12441 \\
(37.783)\end{array}$ & $\begin{array}{l}26.14064 \\
(236.492)\end{array}$ & $\begin{array}{l}2.85382 \\
(5.007)\end{array}$ & 113.018 & 0.05712 & 0.32925 \\
\hline ZBLL & $\begin{array}{c}2.7035 \\
(0.0033)\end{array}$ & $\begin{array}{l}8.16714 \\
(0.823)\end{array}$ & $\begin{array}{c}1.45705 \\
(0.13)\end{array}$ & & & 140.08 & 0.10167 & 0.53337 \\
\hline GL & $\begin{array}{c}67.73728 \\
(29.078)\end{array}$ & $\begin{array}{l}2.76071 \\
(4.312)\end{array}$ & $\begin{array}{l}50.15703 \\
(36.5936)\end{array}$ & & & 112.922 & 0.05965 & 0.33634 \\
\hline
\end{tabular}




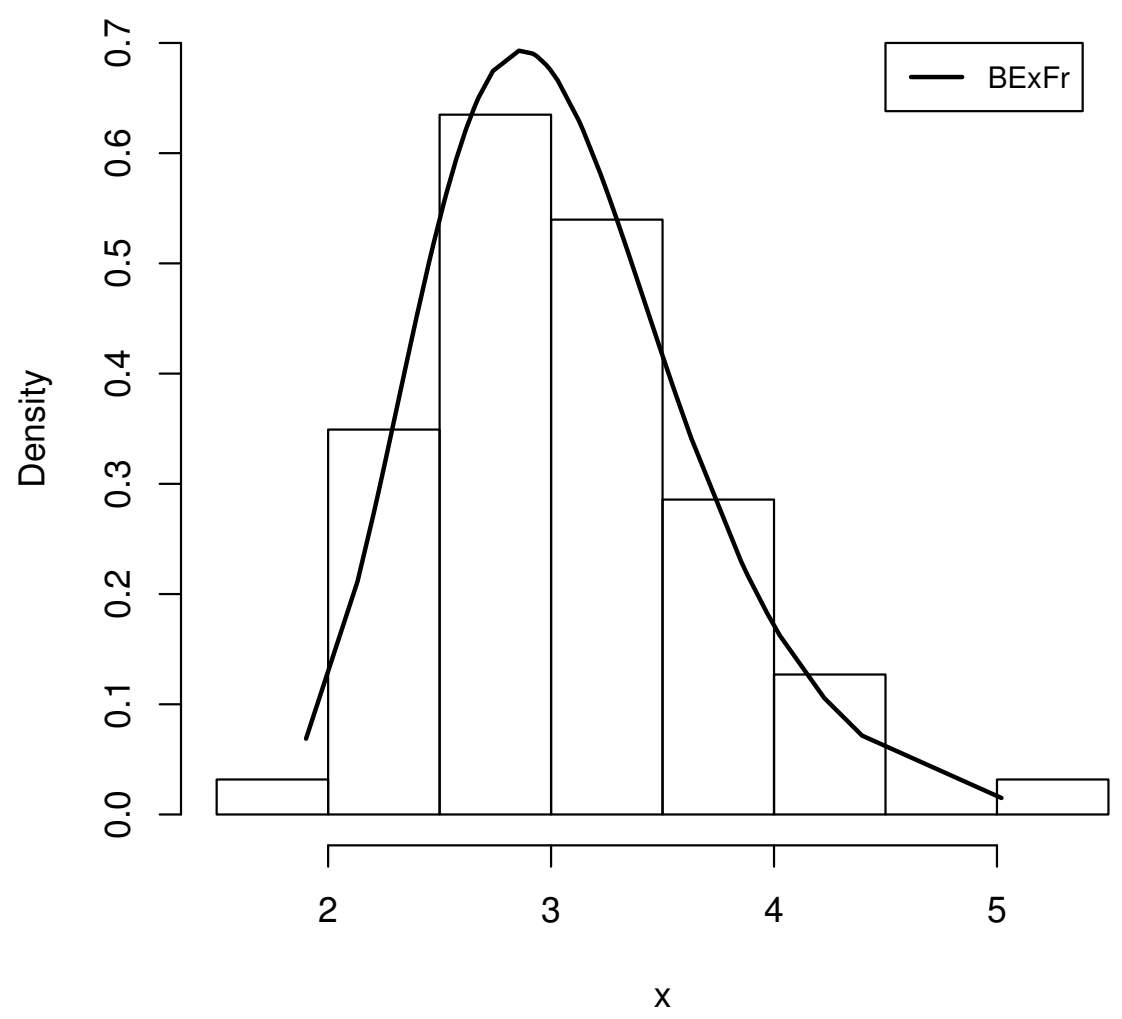

Figure 4: The fitted BExFr density for the second data set. 


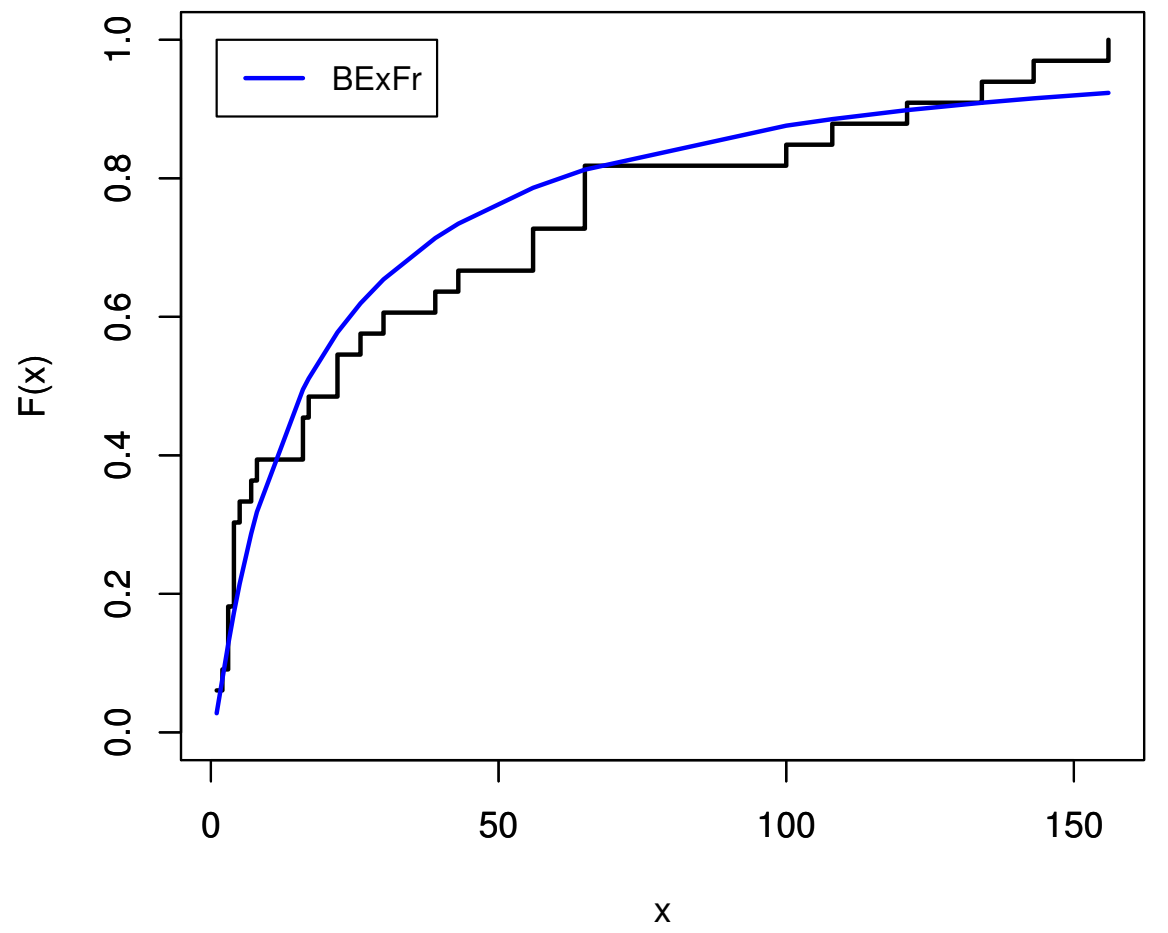

Figure 5: The estimated cdf of the BExFr model for the first data set.

\section{Concluding remarks}

In this paper, we propose a new five-parameter model, called the beta exponential Fréchet (BExFr) distribution, which extends the Fréchet distribution. In fact, the BExFr distribution is motivated by the wide use of the Fréchet distribution in extreme value theory and also for the fact that the generalization provides more flexibility to analyze real data. The BExFr density function can be expressed as a mixture of Fréchet densities. We derive explicit expressions for the ordinary and incomplete moments, moment generating function, entropies, mean residual life and mean inactivity time. We discuss the maximum likelihood estimation of the model parameters. Two applications illustrate that the proposed model provides consistently better fit than the other competitive models.

Estimation of the model parameters under the bayesian paradigm is currently underway and will be reported in a separate article elsewhere. However, we must make a note of the fact under the Bayesian setting, a non informative prior approach is essentially maximum likelihood estimation under the classical approach. In the absence of an appropriate conjugate prior, the choice of prior will be a challenging in such a setting.

As a future work we will consider the following:

- Bivariate and multivariate extension of the BExFr distribution. In particular with the copula based construction method, trivariate reduction etc.

- Comparison of the derived models with the available popular bivariate beta- $G$ and bivariate exponential type models. 


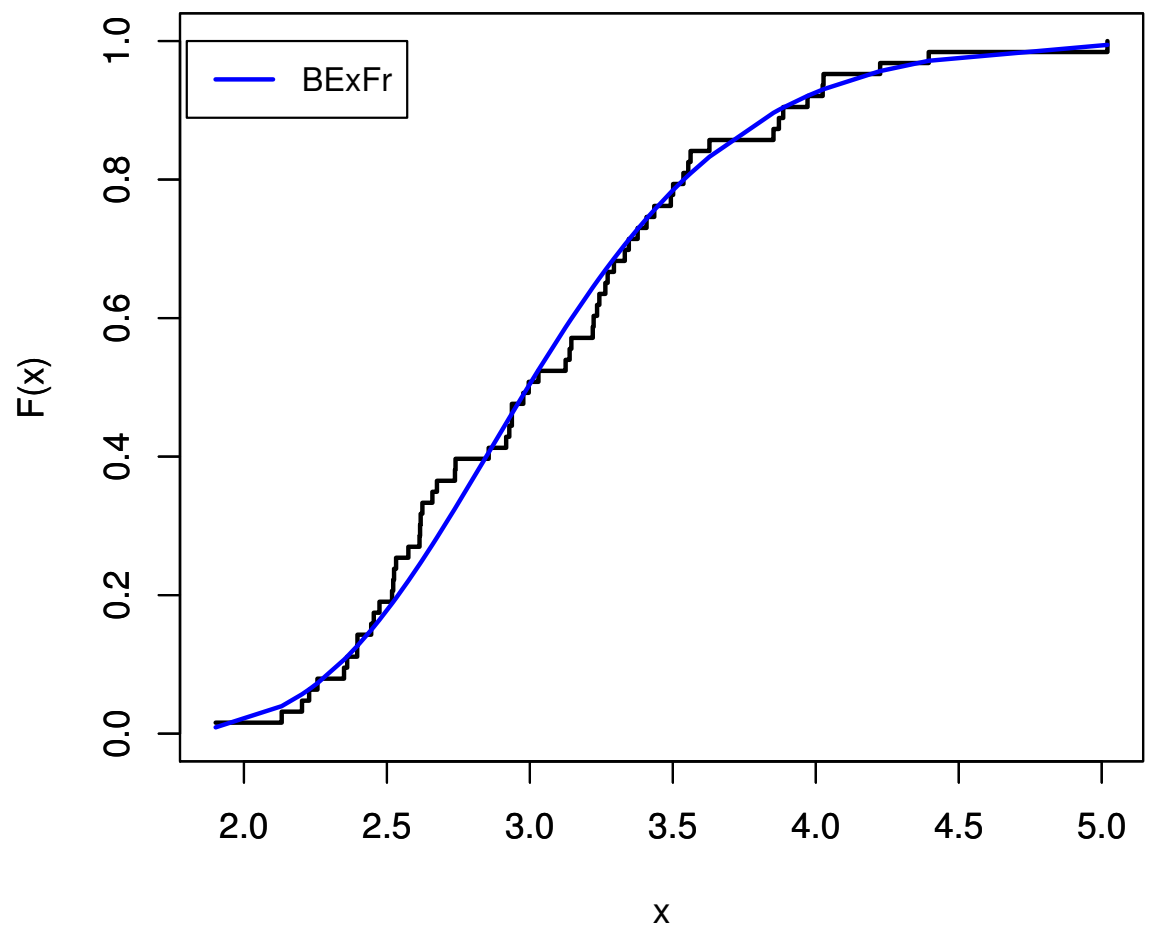

Figure 6: The estimated cdf of the BExFr model for the second data set. 


\section{Acknowledgment}

The authors would like to thank the editor and anonymous referees for their valuable comments and suggestions that improved the quality of the paper.

\section{References}

Agresti, A. (1984). Analysis of Ordinal Categorical Data. New York: John Wiley.

Afify, A.Z., Hamedani, G.G., Ghosh, I. and Mead M.E. (2015a). The Transmuted Marshall-Olkin Fréchet Distribution: Properties and Applications. International Journal of Statistics and Probability, 4, 132-148.

Afify, A.Z., Nofal, Z.M. and Ebraheim, A.N. (2015a). Exponentiated Transmuted Generalized Rayleigh Distribution: A New Four Parameter Rayleigh Distribution. Pakistan Journal of Statistics and Operation Research, 11, 115-134.

Afify, A.Z., Nofal, Z.M., Yousof, H.M., El Gebaly, Y.M. and Butt, N.S. (2015b). The Transmuted Weibull Lomax Distribution: Properties and Application. Pakistan Journal of Statistics and Operation Research, 11, 135-152.

Afify, A.Z., Yousof, H.M., Nofal, Z.M. and Cordeiro, G.M. (2015). The Weibull Fréchet Distribution and Its Applications. Journal of Applied Statistics (Forthcoming.)

Alzaatreh, A., Famoye, F. and Lee, C. (2013). A New Method for Generating Families of Continuous Distributions. Metron, 71, 63-79.

Arnold, B.C. and Laguna, L. (1976). A Stochastic Mechanism leading to Asymptotically Paretian Distributions. Proceedings of the American Statistical Association. Business and Economic Statistics section.

Badar, M.G. and Priest, A.M. (1982). Statistical Aspects of Fiber and Bundle Strength in Hybrid Composites. In: Hayashi, T., Kawata, K., Umekawa, S. (Eds.). Progress in Science and Engineering Composites. ICCM-IV, Tokyo, 1129-1136.

Baharith, L.A., Mousa, S.A., Atallah, M.A. and Elgayar, S.H. (2014). The Beta Generalized Inverse Weibull Distribution. British Journal of Mathematics $\&$ Computer Science, 4, 252-270.

Barreto-Souza, W.M., Cordeiro, G.M. and Simas, A.B. (2011). Some Results for Beta Fréchet Distribution. Communications in Statistics-Theory and Methods, 40, 798-811.

Chen, G. and Balakrishnan, N. (1995). A General Purpose Approximate Goodness-of-fit Test. Journal of Quality Technology, 27, 154-161.

Cordeiro, G.M., Ortega, E.M.M. and Popovic, B.V. (2015). The Gamma-Lomax Distribution. Journal of Statistical computation and Simulation, 85, 305-319.

De Gusmao F.R.S., Ortega E.M.M., and Cordeiro G.M. (2011). The Generalized Inverse Weibull Distribution. Statistical Papers, 52, 591-619.

Elbatal, I., Asha, G. and Raja, V. (2014). Transmuted Exponentiated Fréchet Distribution: Properties and Applications. Journal of Statistics Applications 83 Probability, 3, 379-394.

Feigl, P. and Zelen, M. (1965). Estimation of Exponential Probabilities with Concomitant Information. Biometrics, 21, 826-838. 
Fréchet, M. (1924). Sur la Loi des Erreurs d'Observation. Bulletin de la Société Mathématique de Moscou, 33, 5-8.

Glänzel, W. (1987). A Characterization Theorem Based on Truncated Moments and Its Application to Some Distribution Families. Mathematical Statistics and Probability Theory (Bad Tatzmannsdorf, 1986), B, Reidel, Dordrecht, 75-84.

Guess, F. and Proschan, F. (1988). Mean Residual Life, Theory and Applications. In: Krishnaiah, P.R., Rao, C.R. Handbook of Statistics, Reliability and Quality Control, 7, $215-224$.

Hamada, M. and Wu C.F.J. (1990). A Critical look at Accululation Analysis and Related Methods (with discussion). Technometrics, 28, 302-306.

Keller, A.Z. and Kamath, A.R. (1982). Reliability Analysis of CNC Machine Tools. Reliability Engineering, 3, 449-473.

Kobayashi, K . (1991). On Generalized Gamma Functions Occurring in Diffraction Theory. Journal Physical Society of Japan, 60, 1501-1512.

Kotz, S. and Nadarajah, S. (2000). Extreme Value Distributions: Theory and Applications. Imperial College Press, London.

Krishna, E., Jose, K.K., Alice, T. and Ristic, M.M. (2013). The Marshall-Olkin Fréchet Distribution. Communications in Statistics-Theory and Methods, 42, 4091-4107.

Lai, C.D., and Xie, M. (2006). Stochastic Ageing and Dependence for Reliability. Springer Science and Business Media.

Lemonte, A.J. and Cordeiro, G.M. (2013). An Extended Lomax Distribution. Statistics, 47, 800-816.

Mahmoud, M.R. and Mandouh, R.M. (2013). On the Transmuted Fréchet Distribution. Journal of Applied Sciences Research, 9, 5553-5561.

McCullagh, P. (1980). Regression Models for Ordinal Data (with discussion). Journal of Royal Statistical Society, Series B, 42, 109-142.

Mead, M.E. (2015). Generalized Inverse Gamma Distribution and Its Application in Reliability. Communications in Statistics - Theory and Methods, 44, 1426-1435.

Mead, M.E. and Abd-Eltawab A.R. (2014). A Note on Kumaraswamy-Fréchet Distribution. Australian Journal of Basic and Applied Sciences, 8, 294-300.

Nadarajah, S. and Gupta, A.K. (2004). The Beta Fréchet Distribution. Far East Journal of Theoretical Statistics, 14, 15-24.

Nadarajah, S. and Kotz, S. (2003). The Exponentiated Fréchet Distribution. InterStat. Available online at http://interstat.statjournals.net/YEAR/2003/abstracts/0312001.pdf.

Taguchi G. (1986). Introduction to Quality Engineering. White Plains, NY: Kraus International Publications.

Treyer, V.N. (1964). Doklady Acad, Nauk, Belorus, U.S.S.R.

Zografos, K. and Balakrishnan, N. (2009). On Families of Beta- and Generalized Gammagenerated Distributions and Associated Inference. Statistical Methodology, 6, 344-362. 


\section{Affiliation:}

M. E. Mead

Department of Statistics, Mathematics and Insurance

Zagazig University, Egypt

E-mail: mead9990@gmail.com

Ahmed Z. Afify

Department of Statistics, Mathematics and Insurance

Benha University, Egypt

E-mail: ahmed.afify@fcom.bu.edu.eg

G. G. Hamedani

Department of Mathematics, Statistics and Computer Science

Marquette University, USA

E-mail: gholamhoss.hamedani@marquette.edu

Indranil Ghosh

Department of Mathematics and Statistics

University of North Carolina Wilmington, USA

E-mail: ghoshi@uncw.edu

\section{Austrian Journal of Statistics}

published by the Austrian Society of Statistics

Volume 46

February 2017 http://www.ajs.or.at/

http://www.osg.or.at/

Submitted: 2015-11-01

Accepted: 2016-02-16 